\title{
Aportaciones a las ciencias de la salud: El comportamiento peatonal arriesgado de chilenos y brasileros
}

\section{Contributions to the health sciences: The risky pedestrian behavior of chileans and brazilians}

\author{
Emilio Moyano-Díaz \\ Facultad de Psicología, Universidad de Talca, Chile \\ Renata Jucksch Torquato \\ Alessandra Bianchi \\ Departamento de Psicología, Universidade Federal do Paraná, Brasil
}

(Rec: 03 octubre 2013 / Acept: 08 septiembre 2014)

\begin{abstract}
Resumen
Los peatones son causantes y víctimas de accidentes de tránsito en el mundo y muy especialmente en América latina. Un modelo de comportamiento riesgoso en conductores es aplicado a peatones en dos países del Cono Sur de América: Brasil con la mayor tasa de mortalidad por accidentes de tránsito, y Chile, la menor. Una muestra accidental -463 participantes, 223 chilenos y 240 brasileros, de 17 a 40 años de edad- respondió la Escala de Comportamiento Peatonal controlándose sexo, edad, licencia de conducir y participación en accidentes de tránsito. Contribuyendo a explicar la contrastante mortalidad, los chilenos realizan significativamente menos transgresiones, ligeramente menos errores y lapsus al transitar por la ciudad, que los brasileños. Los hombres de ambos países son más transgresores que las mujeres. Se discute la utilidad del modelo utilizado y de los resultados, entregándose algunas recomendaciones para la salud pública en este ámbito.

Palabras clave: comportamiento peatonal, transgresión y salud pública.
\end{abstract}

\begin{abstract}
Pedestrians are causative and victims of traffic accidents in the world and very especially in Latin America. A model originally built for drivers risk behavior is applied to pedestrians in two contrasting countries in the Southern Cone of America: Brazil has the highest mortality rate for traffic accidents and Chile the lowest. An accidental sample - 463 participants, 223 Chileans and 240 Brazilians of 17 to 40 years of age-, answered an instrument containing the scale of pedestrian behavior (ECP, 1999) and information relating to sex, age, licensed drivers and participation in traffic accidents was collected. Contributing to explain the contrasting mortality, Chileans reported to perform significantly less pedestrian's transgressions, fewer errors and lapses than Brazilian pedestrians. Men in both countries are more transgressors than women. We discuss the utility
\end{abstract}

Correspondencia:

Emilio Moyano-Díaz, Universidad de Talca, Facultad de Psicología, Casilla N 747, Talca/Chile, e-mail: emoyano@utalca.cl, Tel: (5671) 2201713, Fax: (5671) 2201510

Agradecimientos: al Programa de Investigación en Calidad de Vida y Ambientes Saludables de la Facultad de Psicología de la Universidad de Talca (Chile), y al Programa de Mestrado em Psicología da Universidade do Paraná (Brasil). 
and the results of the model used giving some recommendations for public health in this area.

Keywords: pedestrian behavior, transgression, public health.

\section{Introducción}

Los accidentes de tránsito son una causa prevenible de muerte, lesiones y discapacidad. El objetivo del Decenio de Acción para la Seguridad Vial (2011-2020) es estabilizar y después reducir la tendencia al aumento de las muertes por accidentes de tránsito, con lo cual ha sido calculado que se salvarían 5 millones de vidas en esos 10 años (Organización Mundial de la Salud, la OMS, 2013). Según, los accidentes de tránsito cuestan a los países en desarrollo entre el 1 y $2 \%$ de su producto interno bruto (Peden, Scurfield, Sleet, Mohan, Hyder, Jarawan y Mathers, 2004), constituyendo un flagelo desde el punto de vista de la salud pública por la morbi-mortalidad y el sufrimiento involucrados. A diferencia del mundo europeo desarrollado -Gran Bretaña, Países Bajos, Suecia y otros-, en Latinoamérica más del $40 \%$ de las víctimas de tránsito son peatones (ISEV, 2008; OMS, 2009) .

Ha sido estimado que entre el 90 y $95 \%$ de los accidentes de tránsito tienen como único responsable al componente humano del sistema de tránsito (Rumar, 1985), y la literatura psicológica ha estado históricamente más enfocada al estudio de conductores que al de peatones (Moyano-Díaz, 1999; De Winter y Doduo, 2010; Porter, 2011). Así, los enfoques teóricos, los métodos o dispositivos de análisis, y las variables consideradas en la investigación de ambos actores en el tránsito han seguido vías típicamente independientes. Llama la atención que hasta ahora no se utilicen o transfieran, más que excepcionalmente -en lo mejor de nuestro conocimiento-, los desarrollos de la investigación en conductores en beneficio de la investigación y prevención de accidentes en peatones, por lo que en el presente estudio se propone avanzar en ello. Un enfoque, dentro del cual se ubica a numerosos estudios de conocimiento avanzado sobre conductores, corresponde al modelo de Manchester, devenido clásico, referido al comportamiento de riesgo al conducir (Reason, Manstead, Stradling, Baxter y Campbell, 1990; Parker, Manstead, Stradling y Reason, 1992). En él se distingue entre tres tipos de comportamiento de riesgo, que corresponderían a mecanismos psicológicos distintos: violaciones o transgresiones, errores y lapsus. En el modelo se ha entendido por 'violación o transgresión' cualquier acción consciente o deliberada de romper una regla de tránsito para alcanzar un objetivo de tránsito. Se trata de comportamientos de alto riesgo para la salud. Por 'error' se entiende el fracaso de acciones o de comportamientos planeados para la realización de una maniobra de tránsito, a causa de un funcionamiento inapropiado ya sea de mecanismos atencionales, perceptivos o de tratamiento de la información, mientras que por 'lapsus' se entiende una acción de tránsito inadvertida que no era la deseada, no era intencionada (Reason et al., 1990). Esta tipología de comportamientos sirvió de base para la construcción del Driving Behavior Questionnaire (DBQ) (Reason, Manstead, Stradling, Baxter y Campbell, 1990), considerado hoy como el instrumento de auto-reporte más ampliamente utilizado en el mundo, cuya popularidad también proviene de su capacidad para predecir la implicación en accidentes (Wahlberg y Dorn, 2012).

El DBQ ha permitido caracterizar el comportamiento de conductores en y entre diferentes países y culturas, como en Australia (Blockey y Hartley, 1995), Suecia (Aberg y Rimmo, 1998), Brasil (Bianchi y Summala, 2002), Finlandia, Holanda y UK (Lajunen, Parker y Summala, 2004), España (Gras, Sulman Cunill, Planes, Aymerich y Font-Mayolas, 2006), y Emiratos Árabes Unidos (Bener, Ozkan y Lajunen, 2008), entre otros. En estos estudios son controladas las variables sexo y edad y, ocasionalmente, la experiencia en accidentes de tránsito y la exposición. Estudios meta-analíticos con el DBQ han mostrado que las transgresiones y los errores predicen accidentes ( $r=0.13$ y $r=0.10$, respectivamente), donde ambas dimensiones correlacionan negativamente con la edad y positivamente con la exposición (De Winter y Doduo, 2010). Sin perjuicio de esto, en algunas culturas el DBQ no muestra casi diferencias por sexo (Qatar y Emiratos Árabes Unidos), y ha sido sugerido que factores ambientales (culturales), como el contexto de tránsito, carencia de controles y congestión, pudieran ser más importantes que el sexo o factores psicológicos tales como los procesos cognitivos de atención, perceptuales o de procesamiento de la información (Bener, Ozkan y Lajunen, 2008).

No obstante esto, el BDQ se ha mantenido como un instrumento sustancial y útil por más de dos décadas, inspirando nuevos instrumentos para otros actores o poblaciones. Así, existen adaptaciones para ciclistas (Feenstra, Ruiter, Schepers, Peters y Kok, 2011), para motoristas (Elliot, Baughan y Sexton, 2007) y, la más actual, para ambientes laborales (Newman y Von Schckmann, 2012). En consecuencia, el modelo de comportamiento de riesgo en conductores de Reason et al. (1990) tiene un potencial enorme para su aplicación al estudio del comportamiento peatonal, ya que permite objetivar, medir y describir sistemáticamente, transgresiones, errores y lapsus al transitar por la ciudad. 
A partir de esta línea de investigación (Reason et al., 1990) y particularmente del modelo trifactorial propuesto, fue elaborada la escala de comportamiento peatonal (ECP) (Moyano- Díaz, 1999; Díaz, 2002), recientemente adaptada al portugués (Torquato y Bianchi, 2010). Ella permite registrar la frecuencia y clasificar el comportamiento peatonal cotidiano al transitar por la ciudad sobre la base de las mismas tres dimensiones usadas para conductores: transgresiones, errores y lapsus. Algunas aplicaciones a muestras de dos diferentes ciudades chilenas, -Santiago- (Díaz, 2002) y Talca (Moyano-Díaz y Catalán, 2010), mostraron resultados relativos a edad y sexo convergentes con los reportados para conductores. En éstos y en otros estudios, los hombres y los peatones jóvenes reportan realizar más frecuentemente comportamientos transgresores que las mujeres y los adultos (Granié, 2009; Tom y Granié, 2011) o de error (Moyano, Díaz y Catalán, 2010).

En el presente estudio describiremos el comportamiento de peatones, mediante la ECP, en dos países del Cono Sur de América analizando su relación con sexo, edad y accidentes auto-reportados. Hemos elegido Chile y Brasil porque tienen cifras altamente contrastantes de tasas de mortalidad por tránsito. El primero cuenta con la más baja y el segundo con la más alta en la región. Así, Chile presenta 12.3 fallecidos por 100.000 habitantes y Brasil casi el doble: 22.5 por 100 mil (OMS, 2013a). Ambos países comparten una semejante alta mortalidad en atropello de peatones, la que ocupa el primer lugar después de las muertes por colisiones de vehículos en conductores y pasajeros (Comisión Nacional de Seguridad Tránsito, CONASET, 2008), y se desconoce qué podría explicarla.

La tasa de muertes por accidente de tránsito en Chile se estabilizó entre los años 2006 a 2009 en alrededor de 12/100.000 hbts., en tanto para Brasil ella se estabilizó de 2004 a 2009 en alrededor de 20/100.000 (OMS, 2013a). Los dos países también tienen una tasa de motorización relativamente similar, 19.4 vehículos cada 1000 habitantes para Chile y 17.7 para Brasil, con 4.9 personas por vehículo en Chile y 4.2 en Brasil (Asociación Latinoamericana de Distribuidores de Automotores, ALADDA, 2012). Más globalmente, ambos países tienen economías emergentes y ciudades con crecientes problemas relativos al tránsito (y al consumo de energía y manejo de basuras) (Mosler y Kraemer-Palacios, 2012). A causa de la contrastante tasa de mortalidad por tránsito entre ambos países, en el presente estudio se espera hallar más transgresiones, más errores y más lapsus en la muestra brasilera que en la chilena, lo que constituye nuestra hipótesis general de trabajo. También y más específicamente, se espera encontrar diferencias por sexo -hombres con más transgresiones que mujeres-, por edad -adultos jóvenes más transgresores y con más comportamientos erróneos que adultos mayores-, tanto para Chile como para Brasil, y que ello aparezca asociado a accidentes, de modo convergente con los resultados la literatura relativa a conductores. Adicionalmente, del conjunto de resultados derivaremos algunas recomendaciones para campañas de prevención en seguridad de tránsito y salud pública.

\section{Método}

\section{Muestra}

Extraída de modo accidental y constituida por 463 ciudadanos de ambos sexos (256 mujeres y 207 hombres), entre 17 y 40 años (302 jóvenes de 17 a 25 años, y 161 adultos de 26 en adelante). Los chilenos (de Talca, 223) con edad media de 28 años $(D T=6.12)$ y los brasileros (de Curitiba, 240) de 21 años de media $(D T=4.37)$.

\section{Instrumentos}

Escala de Comportamiento Peatonal (ECP) de MoyanoDíaz (1999) con 17 ítems referidos a comportamientos transgresores, de error y de lapsus, que se responden en formato Likert desde 1 (nunca) a 6 (siempre). En el presente estudio, la ECP, en sus versiones original castellana chilena (Moyano-Díaz, 1999; Díaz, 2002) y adaptación portuguesa brasilera (Torquato y Bianchi, 2010), presentó una buena confiabilidad para ambas muestras (alfa $=.85$ y .81 Chile y Brasil, respectivamente). Se solicitó también información socio-demográfica del respondiente: edad, sexo, ocupación, si ha sufrido accidente(s) de tránsito y en qué número, $\mathrm{y}$ si dispone de licencia de conducción o no. Si dispone de ella y conduce regularmente fue considerado aquí como chofer para efectos de la conformación de subgrupos de comparación, mientras que si no disponía de ella ni conducía regularmente fue considerado como peatón.

\section{Procedimiento}

Se solicitó a grupos de estudiantes universitarios en salas de clase completar la ECP, previa firma de un documento de consentimiento informado. Los participantes no estudiantes fueron abordados individualmente en estacionamientos de supermercados y plazas públicas de ambas ciudades, sometiéndoles el mismo protocolo de consentimiento informado. Para el análisis estadístico se verificó los criterios relativos 
a normalidad y homocedasticidad de los datos, y posteriormente fueron calculados los promedios de frecuencia de los comportamientos consignados en la ECP, y sus diferencias empleando t test.

\section{Resultados}

En primer lugar, y como se Tabla 1 para la muestra total de participantes, se puede afirmar que éstos realizan, con una frecuencia que se ubica entre intermedia y moderada -valores promedio alrededor de 3 y superiores sobre 6-, comportamientos peatonales de transgresión, de error y de lapsus al transitar cotidianamente en sus ciudades. Descriptivamente, el reporte de los tres tipos de comportamiento de riesgo muestra una frecuencia promedio menor para transgresiones (entre 2.0 y 2.9 ) que para errores y lapsus (mayoritariamente entre 3.0 y 3.5 ).

A continuación se reportarán resultados relativos a las variables sexo y edad, y las respuestas generales a los instrumentos por cada país. Se seguirá con los resultados referidos a los tres tipos de comportamiento de riesgo del modelo -transgresor, de error y de lapsus-, sus semejanzas y diferencias para los participantes de ambos países, para posteriormente realizar algunas conclusiones y recomendaciones para campañas de seguridad de tránsito.

Antes de aplicar la ECP en Brasil, se siguieron las sugerencias de la ITC (2010) sobre traducción y adaptación de tests. Con el objetivo de validar el factor de comportamiento arriesgado se realizó un anáisis factorial para la muestra chilena y la brasilera separadamente, obteniéndose una estructura similar.
En ambas muestras, los indicadores promediados de los tres tipos de comportamientos (transgresiones, lapsus y errores) apoyaron una sola dimensión, al igual que en publicaciones anteriores (Díaz, 2002). Esta solución unifactorial muestra porcentajes de varianza explicada superiores a .60 (68.21\% y $62.96 \%$ ). Para determinar este factor se seleccionó el método de extracción de componentes principales, aplicando además una rotación varimax. En ambos casos, los estadísticos KMO de adecuación muestral arrojaron valores óptimos (.644 y .676). Para determinar la unidimensionalidad factorial de la estructura latente se siguieron tres criterios: a) la regla $\mathrm{K} 1$ (Kaiser, 1960), combinada con b) el gráfico de sedimentación de los autovalores (Cattell, 1966), y c) un análisis paralelo (Horn, 1965). En primer lugar, hubo solo un componente con un valor superior a 1. En segundo lugar, el gráfico de sedimentación mostró claramente cómo el número y tamaño de autovalores del análisis cambiaban su pendiente por debajo de 1, para dar paso a una planicie de factores residuales. Y, en tercer lugar, el análisis paralelo indicó solo un autovalor mayor que los estimados aleatorios (media y $>\mathrm{C}_{95}$ ).

Comparación de las muestras por tipo de comportamientos. La frecuencia de comportamientos de transgresión, error y lapsus sigue un patrón similar en ambos países, por cuanto los comportamientos del tipo error aparecen como modales, $\mathrm{y}$, aunque son de frecuencia relativamente más alta para los peatones chilenos, esta diferencia no es significativa. Solo se observa una diferencia pequeña y significativa en los comportamientos transgresores que los participantes brasileros reportaron hacer en mayor medida que los chilenos $(M=2.93$ y $M=2.73$ respectivamente; $t(461)=2.11, p=.012, d=.243)$. En los otros dos tipos

Tabla 1.

Estadísticos descriptivos y tamaño del efecto en las dimensiones de la escala ECP para chilenos ( $n=223)$ y brasileros ( $n=240)$.

\begin{tabular}{lllllll}
\hline \multirow{2}{*}{ ECP } & \multicolumn{1}{l}{ Chile } & & Brasil & \\
\cline { 2 - 3 } & Media & DT & & Media & DT & $d$ \\
\hline Transgresión & 2.73 & 0.85 & & 2.93 & 0.80 & .243 \\
Error & 3.34 & 1.08 & & 3.38 & 1.04 & .038 \\
Lapsus & 2.98 & 1.09 & & 3.14 & 1.15 & .143 \\
\hline
\end{tabular}

Nota. rango de la escala: 1 (nunca) a 6 (siempre). En negrita los tamaños de efecto entre las medias que también mostraron diferencias significativas. 
Tabla 2.

Promedios para cada tipo de comportamiento peatonal y significación de diferencias $(t)$ para subgrupos de chilenos $(n=223)$ y brasileros $(n=240)$.

\begin{tabular}{|c|c|c|c|c|c|c|c|c|c|c|c|c|c|c|c|c|}
\hline \multirow{2}{*}{ País } & \multirow{2}{*}{ ECP } & \multicolumn{2}{|c|}{ Jóvenes } & \multicolumn{3}{|c|}{ Adultos } & \multicolumn{2}{|c|}{ Hombres } & \multicolumn{3}{|c|}{ Mujeres } & \multicolumn{2}{|c|}{ Conductores } & \multicolumn{3}{|c|}{ Peatones } \\
\hline & & M & DT & M & DT & $\mathrm{D}$ & M & DT & M & DT & d & M & DT & M & DT & d \\
\hline \multirow{4}{*}{ Chile } & Transgresión & 2.86 & 0.93 & 2.65 & 0.78 & .253 & 2.89 & 0.89 & 2.62 & 0.80 & .320 & 2.84 & 0.86 & 2.60 & 0.82 & .278 \\
\hline & Error & 3.47 & 1.15 & 3.25 & 1.04 & .201 & 3.49 & 1.03 & 3.23 & 1.11 & .240 & 3.54 & 1.06 & 3.06 & 1.06 & .455 \\
\hline & Lapsus & 3.02 & 1.15 & 2.95 & 1.03 & .061 & 3.00 & 1.15 & 2.96 & 1.02 & .036 & 3.16 & 1.09 & 2.74 & 1.01 & .397 \\
\hline & Transgresión & 2.98 & 0.79 & 2.55 & 0.78 & .376 & 3.04 & 0.89 & 2.83 & 0.70 & .257 & 3.02 & 0.84 & 2.84 & 0.75 & .220 \\
\hline \multirow[t]{2}{*}{ Brasil } & Error & 3.45 & 1.03 & 2.84 & 0.91 & .413 & 3.33 & 1.00 & 3.41 & 1.07 & .082 & 3.40 & 1.10 & 3.35 & 0.98 & .051 \\
\hline & Lapsus & 3.17 & 1.16 & 2.93 & 1.05 & .145 & 2.90 & 1.16 & 3.36 & 1.10 & .410 & 3.11 & 1.22 & 3.16 & 1.06 & .047 \\
\hline
\end{tabular}

Nota: rango de la escala: 1(nunca) a 6 (siempre). En negrita los tamaños de efecto entre las medias que también mostraron diferencias significativas.

de comportamiento de la ECP no se encontró diferencias significativas.

Los promedios para las tres dimensiones de comportamiento de la ECP presentan en las dos muestras una misma configuración, con un mismo orden decreciente de valores, respectivamente, para error, lapsus y transgresión en los participantes chilenos y brasileros. La comparación en Chile entre peatones y choferes muestra que los primeros cometen más frecuentemente comportamientos trasgresores, de lapsus y de error al transitar por la ciudad $(t(211)=$ $2.05, p=.042, d=.278 ; t(221)=3.35, p<.000, d=.455$; $t(221)=2.92, p=.004, d=.397)$. Respecto de la variable edad, para Chile no se registran diferencias significativas en transgresión sino una tendencia $(t(221)=1.86, p=.065$, $d=.253$ ), con valor más alto en la ECP para los jóvenes, pero sí para Brasil donde los jóvenes ejecutan más comportamientos transgresores y de error que los adultos en su tránsito habitual (ver Tabla 2).

Se observan también diferencias en cuanto al comportamiento trasgresor al transitar por la ciudad según rol en el ambiente de tránsito (peatón/chofer) y sexo de los participantes (Tabla 2) para ambas muestras, donde los peatones y los hombres poseen más comportamiento transgresor que los choferes y mujeres respectivamente. No se observan diferencias según sexo ni edad en lo que respecta a comportamientos de lapsus y de error, en chilenos, aunque sí respecto del rol, ya que los peatones los cometen significativamente más que los choferes.

Para la muestra de Brasil, se observa a los hombres reportando más comportamiento transgresor que las mujeres, y a éstas teniendo más comportamientos de lapsus que aquéllos $(t(238)=1.98, p=.049, d=.257 ; t(238)=3.15, p=.002, d$ $=.410)$, sin observarse diferencias significativas en cuanto a errores. Respecto del rol en el ambiente de tránsito, los choferes reportan un poco más de transgresiones que los peatones $(t(237)=1.69, p=.092, d=.220)$ (inversamente que para chilenos), sin registrarse tampoco diferencias en cuanto a comportamientos de error ni de lapsus entre ambos. En lo que respecta a edad, los jóvenes presentan más transgresiones y errores al transitar por la ciudad que los adultos $(t(238)=2.75, p=.007, d=.376 ; t(238)=3.02$ $p=.003, d=.413)$, sin observarse diferencias respecto a lapsus (Tabla 2).

Finalmente, haber experimentado accidentes de tránsito previos no tiene ningún efecto diferenciador en cuanto al despliegue de alguno de los tres tipos de comportamiento aquí analizados, ni para chilenos ni para brasileros.

\section{Conclusiones y discusión}

El modelo de tres tipos de comportamiento de riesgo en el tránsito de conductores resulta una herramienta conceptual y empíricamente útil para objetivar, medir y diferenciar el comportamiento de peatones en Chile y Brasil. Así, la ECP permite capturar similitudes y diferencias en el comportamiento peatonal en estos dos países y al interior de cada uno. Globalmente, lo más interesante es que la principal diferencia encontrada entre ambas muestras -el comportamiento transgresor, el de mayor riesgo para la salud-es claramente consistente con la prevalencia de mayor accidentalidad y mortalidad en Brasil que en Chile. Ello aumenta nuestra comprensión respecto de los comportamientos de riesgo que están a la base de esas contrastantes tasas de mortalidad por accidentes de tránsito entre los dos países.

Para la muestra total de participantes de este estudio -Chile y Brasil- se observa que los jóvenes de ambos sexos y los hombres adultos cometen más transgresiones y errores en su comportamiento de tránsito habitual que los adultos y mujeres, lo que es convergente con el hecho de que corresponden a grupos de mayor siniestralidad según las estadísticas nacionales en estos dos países, y también 
a las internacionales en el ámbito. Este primer resultado general contribuye a explicar por qué los miembros de estos subgrupos etarios y sexuales son las principales víctimas -y quizá simultáneamente provocadores- de accidentes. En consecuencia, estos subgrupos debieran ser blancos de programas de salud pública referidos a educación de tránsito, no solo respecto de prevención de transgresiones flagrantes a las reglas de tránsito (como ocasionalmente se hace), sino que también respecto de comportamientos erróneos que por definición no están motivados consciente o deliberadamente, y que más bien corresponden a una inapropiada estimación de las condiciones particulares o específicas del ambiente de tránsito en un momento dado, y que resulta en accidente.

Por cierto, la comisión de errores y de transgresión en el comportamiento peatonal de los jóvenes puede ser vinculado a fenómenos que les son característicos, como una elevada percepción de invulnerabilidad, optimismo ilusorio y sobrestimación del propio autocontrol (MoyanoDíaz y Catalán, 2010). Sin embargo, no es tan claro que estos factores sirvan para explicarlo en los adultos. La relativamente alta comisión de errores en los adultos es convergente con su creciente mayor participación en la estadística de mortalidad, ya que ella va aumentando progresivamente conforme la edad, hasta alcanzar en el tramo de 46 años en adelante el primer lugar en mortalidad por tránsito en Chile. Este hecho es contra-intuitivo, toda vez que se trata de personas que por su edad podemos considerar como experimentadas en circulación de tránsito, es decir, como poseedoras de comportamientos saludables y de auto-cuidado ya largamente instalados en su repertorio habitual. Tal vez esto se pueda explicar evolutivamente. Quizás en su juventud estas personas adquirieron ciertas destrezas para desplazarse peatonalmente con seguridad, realizando incluso comportamientos riesgosos de transgresión, erróneos y de lapsus (cruzar calles en momentos o lugares no permitidos, etcétera) en situaciones de tránsito complejas, pero sin consecuencias negativas gracias a un buen estado físico, de reflejos y de salud general, propios de la edad. La manutención de estas acciones como patrón de hábitos comportamentales puede ser considerada como una habituación a conductas de riesgo. Ahora, sin embargo, con la progresiva pérdida de reflejos, aumento del tiempo de reacción, sumado a una mayor vulnerabilidad física, les hace quedar más expuestos a ser víctimas de accidentes que antes y con consecuencias de mayor daño por su edad (Sullman et al., 2011).

Respecto del sexo, si bien los hombres son más transgresores que las mujeres, no se diferencian de éstas en cuanto a la frecuencia de errores y lapsus al transitar. Así, si se tratara de hacer campañas para peatones para corregir errores y lapsus al transitar, éstas podrían ser sexualmente indiferenciadas, en cambio si se trata de buscar inhibir comportamientos transgresores, éstas debieran focalizarse en hombres. Estos resultados guardan relación con la conducta peatonal diferenciada que tienen las personas en general (Granié, 2009; Sullman et al., 2011; Tom y Granié, 2011). En Chile los peatones 'puros' (no choferes) cometen más errores al transitar que los choferes y así, además de ser tipificados como 'víctimas' o 'grupo vulnerable'-lo que es habitual a los choferes en nuestros países-, ellos debieran ser re-tipificados en su rol en los accidentes de tránsito como participantes activos en la producción de éstos. En consecuencia, también debieran constituirse en un foco de atención prioritario en educación de tránsito y salud pública. En Brasil, en cambio, al ser los choferes quienes declaran cometer más comportamiento transgresor que los peatones, debieran seguir siendo un blanco prioritario de campañas de prevención en salud o seguridad de tránsito.

Globalmente, la comparación permitió comprobar la hipótesis central que los participantes brasileros presentan cifras más altas de realización de los tres tipos de comportamiento peatonal medidos por la ECP que los chilenos, aunque es solo en la dimensión de transgresión donde la diferencia tiene significación estadística. Si bien los tres tipos de comportamientos pueden representar riesgo en el tránsito, el comportamiento transgresor por definición es el más riesgoso ya que comúnmente consiste en una flagrante ruptura a la armonía del ambiente de tránsito, quebrando la predictibilidad propia de un tránsito seguro. Los participantes brasileros aparecen aquí cometiendo significativamente más transgresiones que los chilenos. Este resultado es consistente al reflejar la situación general de la seguridad de tránsito de ambos países, con más riesgo de mortalidad de tránsito para los brasileros que para los chilenos, y eso aun considerando que al menos en Curitiba, Brasil, se cuenta con un sistema de transporte integrado de alto estándar de seguridad (vías segregadas, vías exclusivas, ciclo-vías, etcétera) respecto del de Talca y de Chile en general. Quizá ese sistema de transporte moderno en Curitiba ejerza un efecto de 'falsa' seguridad o protección a usuarios de las vías, que a causa de ello se tornan más arriesgados, siguiendo la teoría homeostática del riesgo (Wilde, 1982). Una hipótesis derivable de ésta es que los brasileros, al observar y vivir en un ambiente de tránsito cuya infraestructura es más moderna, inteligible y aparentemente más segura, aumentan su comportamiento de riesgo adaptándolo a un nivel tolerable según las condiciones de seguridad percibidas. 
Desde otra perspectiva, las diferencias entre ambas ciudades también podrían estar explicadas por otro factor ambiental agregado, aquel referido a que entornos menos transitados y abundantes áreas verdes tienden a relacionarse con un transitar más lento (Franek y Ondracek, 2010). Tal vez esto podría ser en parte el caso de Talca, ya que presenta un menor desarrollo y tránsito urbano, con una circulación y un estilo de vida más lento que el de Curitiba.

Entre las semejanzas más destacables halladas aquí, los hombres de ambos países cometen significativamente más transgresiones al transitar por sus ciudades como peatones que las mujeres e, igualmente, son los jóvenes quienes las cometen más que los adultos, aunque para los chilenos ello se manifieste solo como tendencia estadística (al 10\%). Estos resultados globalmente convergen con los reportados en la literatura referida a otros comportamientos transgresores y de riesgo de conductores en tránsito (uso de cinturón, alcohol), asociados a accidentes con daños o lesiones (Turner, McClure y Pirozzo, 2004).

Entre los chilenos, los peatones cometen no solo más transgresiones que los choferes sino que también más errores y lapsus que éstos. Aunque los valores obtenidos según edad respecto de la frecuencia de los comportamientos evaluados al transitar tienden a ser superiores para los jóvenes en realación a los adultos, ello no alcanza significación estadística $\mathrm{y}$, acerca del sexo, solo se registra una diferencia -relativa a transgresión- asociada a sexo masculino.

En Brasil, en cambio, son los choferes quienes cometen más transgresiones, los jóvenes más errores que los adultos y las mujeres más lapsus que los hombres. Adicionalmente, los brasileros presentan una mayor diferenciación en las variables. Así, globalmente se podría decir que la muestra chilena parece tener una cultura más homogénea, respecto del comportamiento de tránsito peatonal, que la muestra brasilera. Desde esta perspectiva, se podría considerar esta variabilidad propia del comportamiento de la muestra brasilera en próximos estudios como hipótesis de riesgo de tránsito, toda vez que un comportamiento poblacional más homogéneo en el espacio público resulta más predecible y así más seguro, según un principio básico de seguridad de tránsito.

Acerca de si existe un patrón de comportamiento de riesgo diferenciado entre quienes han experimentado accidentes de tránsito previamente respecto de quienes no, el estudio mostró que no hay diferencias. Esto es así para cualquiera de los tres tipos de comportamiento de riesgo evaluados aquí, resultado que es consistente con el reportado en estudios previos en Chile (Moyano-Díaz y Catalán, 2010). Sin perjuicio de ello, esto genera preguntas sobre la función o efectos de una experiencia de accidente de tránsito encomportamientos de tránsito subsecuentes. Existe alguna literatura que muestra que haber estado involucrado en accidente de tránsito tiene un efecto de moderación conductual posterior solo temporal, relativamente breve, de no más de seis meses, luego del cual las personas regresan a su comportamiento -arriesgado- habitual (Rajalin y Summala, 1997). Adicionalmente, también habría que afinar el instrumento utilizado respecto de la información relativa a accidentes de tránsito, para distinguir con mayor precisión el tiempo transcurrido, la gravedad, el rol protagónico o no, y otras características del evento que pudieran resultar eventualmente diferenciadoras de efectos posibles y la duración de éstos.

Finalmente, creemos que transferir conceptos, modelos e instrumentos que se han probado eficientes en la investigación de la accidentalidad de tránsito de conductores, como hemos hecho aquí con el modelo de comportamiento de riesgo del Grupo de Manchester, resulta muy útil para avanzar en la investigación de estos otros actores fundamentales del componente humano del sistema de tránsito, como son los peatones. Dada la alta mortalidad por tránsito en general -y de peatones en particular- en los países del Cono Sur y más generalmente de América Latina, esto cobra especial relevancia.

\section{Referencias}

Aberg, L. y Rimmo, P. A. (1998). Dimensions of aberrant driver behavior. Ergonomics, 41, 39-56.

Aladda. Tasa de motorización (2011). Recuperado desde www. com/ trabajos/2012/IndiceMotorizacion2011.xls.

Bener, A., Ozkan, T. y Lajunen, T. (2008). The Driver Behaviour in Arab Gulf countries: Qatar and United Arab Emirates. Accident Analysis and Prevention 40, 1411-1417.

Bianchi, A. y Summala, H. (2002). Moral judgment and drivers behavior among Brazilian students. Psychological Reports, 91, 759-766.

Blockey, P. N. y Hartley, L. R. (1995). Aberrant driving behavior: errors and violations. Ergonomics, 38, 1759-1771.

Cattell, R. B. (1966). The Scree Test for the Number of Factors. Multivariate Behavioral Research, 1, 245-276.

CONASET (2008). Atropellos en Chile, año 2007. Secretaría Ejecutiva Comisión Nacional de Seguridad del Tránsito.

De Winter, J. C. F. y Doduo, D. (2010). The Driver Behaviour Questionnaire as a predictor of accidents: A meta-analysis. Journal of Safety Research, 41, 463-470.

Díaz, M. E. (2002). Theory of Planned Behavior and pedestrians' intentions to violate traffic regulations. Transportation Research Part F, 5. Traffic Psychology and Behaviour, 5, 169-175.

Elliott, M. A., Baughan, C. J. y Sexton, B. F. (2007). Errors and violations in relation to motorcyclists' crash risk. Accidents Analysis and Prevention, 39, 491-499.

Feenstra, H., Ruiter, R. A, Schepers, J, Peters, G. J. y Kok, G. (2011). Measuring risky adolescent cycling behaviour. Int J Inj Contr Saf Promot, 18, 181-187.

Franek, M. y Ondracek, L. (2010). Environmental factors influencing pedestrian speed in cities. Ceskoslovenska Psychologie, 54, 455-471. 
Granié, M. A. (2009). Effects of gender, sex-stereotype conformity, age and internalization on risk-taking among adolescent pedestrians. Safety Science, 47, 1277-1283.

Gras, M. E., Sulman, M. J. M., Cunill, M., Planes, M., Aymerich, M. y Font-Mayolas, S. (2006). Spanish drivers and their aberrant driving behaviours. Transport Research Part F, 9, 129-137.

Horn, J. L. (1965). A Rationale and test for the number of factors in factor analysis. Psychometrika, 30, 179-185.

ISEV (2008). Informe latinoamericano ISEV sobre tránsito y seguridad vial. Instituto de Seguridad y Educación Vial. Buenos Aires, Argentina.

International Test Commission (ITC) (2010). International Test Commission Guidelines for Translating and Adapting Tests. Recuperado desde http://www.intestcom.org/guidelines/index.phphttps://exchange.utalca. $\mathrm{cl} /$ owa/redir.aspx?C $=63$ bcd9d108594ce69a72c14a6740ca0d\&URL $=$ ht tp $\% 3 \mathrm{a} \% 2 \mathrm{f} \% 2$ fwww.intestcom.org $\% 2$ fguidelines $\% 2$ findex.php

Kaiser, H. F. (1960). The application of electronic computers to factor analysis. Educational and Psychological Measurement, 20, 141-151.

Lajunen, T., Parker, D. y Summala, H. (2004). The Manchester Driver Behaviour Questionnaire: a cross cultural study. Accident Analysis and Prevention 36, 231- 238 .

Mosler, H. J. y Kraemer-Palacios, S. (2012). Environmental issues in developing countries. En L. Steg, A. E. Van den Berg, y J. I. M. de Groot (Eds), Environmental Psychology: An Introduction (pp.293-302). Oxford, UK: John Wiley y Sons.

Moyano-Díaz, E. (1997). Evaluation of traffic violation behaviors and the causal attribution of accidents in Chile. Environment and Behavior 29, 264-282.

Moyano-Díaz, E. (1999). Psicología Social y seguridad de tránsito. Sello Editorial Universidad de Santiago de Chile. Santiago, Chile.

Moyano-Díaz, E. Catalán, J. (2010). Calidad de vida urbana: contexto epidemiológico de la seguridad de tránsito en Chile y comportamiento peatonal en la región del Maule. E. Moyano-Díaz (Ed.), Calidad de vida y Psicología en el Bicentenario de Chile (pp. 219-248). Santiago: Marmor.

Newnam, S. y Von Schuckmann, C. (2012). Identifyng an appropriate driving behaviour scale for the occupational driving context: The BDQ vs. the ODBQ. Safety Science 50, 1268-1274.

Organización Mundial de la Salud, OMS (2009). Informe sobre la Situación Mundial de la Seguridad Vial: Es hora de pasar a la acción.
Departamento de Prevención de la Violencia y los Traumatismos y Discapacidad (VIP). Ginebra, Suiza.

Organización Mundial de la Salud, OMS (2013). Informe sobre la Situación Mundial de la Seguridad Vial. Departamento de Prevención de la Violencia y los Traumatismos y Discapacidad (VIP). Ginebra, Suiza.

Organización Mundial de la Salud, OMS (2013a). Global status report on road safety 2013. Supporting a decade of action. World Health Organization. Printed in Luxembourg.

Parker, D., Manstead, A. S. R., Stradling, S. G. y Reason, J. T. (1992). Determinants of intention to commit driving violations. Accident Analysis and Prevention, 24, 117-134.

Peden, M., Scurfield, R., Sleet, D., Mohan, D., Hyder, A. A., Jarawan, E. y Mathers, C. (Ed.) (2004). World report on road traffic injury prevention, Geneva: World Health Organization.

Porter, B. E. (2011). Handbook of Traffic Psychology. Academic Press, Elsevier, London, UK.

Rajalin, S. y Summala, H. (1997). What surviving drivers learn from a fatal road accident. Accident Analysis and Prevention, 29, 277-283.

Reason, J., Manstead, A., Stradling, S., Baxter, J.A. y Campbell, K. (1990): "Errors and violations on the road: A real distinction?" Ergonomics, 33, 1315-1332.

Rumar, K. (1985). The rol of perceptual and cognitive filters in observed behaviour. In L. Evans and R. C. Schwing (Eds.), Human Behavior and Traffic Safety (pp. 151-165). New York: Plenum Press.

Stradling, S.G. y Parker, D. (1996). Violations on the road: Bad attitudes make bad drivers. Presented to symposium on "Attitudes and values Concerning Road Safety”. Birmingham. U.K.

Sullman, M. J. M., Gras, M.E., Font-Mayolas, S., Masferrer, L., Cunill, M. y Planes, M. (2011). The pedestrian behaviour of Spanish adolescents. Journal of Adolescence, 34, 531-539

Tom, A. y Granié, M. A. (2011). Gender differences in pedestrian rule compliance and visual search at signalized and unsignalized crossroads. Accident Analysis and Prevention, 43, 1794-1801.

Torquato, R. y Bianchi, A. S. (2010). Comportamento de Risco do Pedestre ao Atravessar a Rua: Um Estudo com Universitários. Transportation: Theory and Application, 2, 19-41.

Turner, C., McClure, R. y Pirozzo, S. (2004). Injury and risk-taking behavior: a systematic review. Accident Analysis and Prevention, 36, 93-101.

Wahlberg, A.E Af., y Dom, L. (2012). Commentaries and Responses to "The Driver Behaviour Questionnaire as a predictor of accidents: A meta-analysis" Commentaries lead by Anders af Wåhlberg; Responses lead by J.C.F. de Winter. Journal of Safety Research, 43, 83-99.

Wilde, G. (1982). The theory of risk homeostasis: Implications for safety and health. Risk Analysis, 2, 209-225. 